\title{
Time associated with intravenous zoledronic acid administration in patients with breast or prostate cancer and bone metastasis
}

This article was published in the following Dove Press journal:

Cancer Management and Research

2I February 2012

Number of times this article has been viewed

\author{
Akshara Richhariya' \\ Yi Qian ${ }^{2}$ \\ Yufan Zhao ${ }^{2}$ \\ Karen Chung'
}

'Amgen Inc, Global Health Economics, Thousand Oaks, CA, USA; ${ }^{2}$ Amgen Inc, Global Biostatistical Sciences, Thousand Oaks, CA, USA
Correspondence: Akshara Richhariya Amgen Inc, Department of Global Health Economics, I Amgen Center Drive, Thousand Oaks, CA 91320, USA

$\mathrm{Tel}+\mathrm{I} 8054473438$

Fax + I 805376 I8I6

Email aksharar@amgen.com
Purpose: Intravenous (IV) zoledronic acid (ZA) is commonly used to delay skeletal complications secondary to bone metastases. However, the time associated with ZA administration may represent a significant burden to healthcare providers and patients. This study assessed the time associated with IV ZA infusion in patients with bone metastases secondary to breast or prostate cancer (BC or PC) in the clinic setting.

Methods: Eligible BC or PC patients with bone metastases scheduled to receive IV ZA were observed at seven US-based oncology clinics. Trained observers recorded the time for preinfusion tasks, ZA drug preparation, intravenous infusion, and follow-up activities.

Results: Data are reported for 39 patients (BC: 24; PC: 15). Mean administration time was 69 (standard deviation [SD] 42) minutes for all patients combined, 72 (SD 47) minutes for BC, and 65 (SD 33) minutes for PC. Activity times were comparable between tumor types. Mean time for preinfusion tasks (eg, assessment of vital signs, blood draw) and ZA preparation were 12 (SD 20) minutes and 2 (SD 1) minutes, respectively. Mean time required for intravenous infusion (ZA infusion and hydration, when provided) and follow-up activities were 54 (SD 31) minutes and 2 (SD 1) minutes, respectively.

Conclusion: Infusion time was the greatest time commitment associated with IV ZA administration, representing $78 \%$ of the total time on average. Time for preinfusion activities varied substantially. Overall, the mean time for ZA administration represents a notable time burden for healthcare providers and patients.

Keywords: time and motion, bisphosphonates, zoledronic acid, intravenous administration

\section{Introduction}

The diagnosis of bone metastases represents a sentinel event in the progression of solid tumors. The goal of treatment in patients with bone metastases is to prevent or delay skeletal complications, maintain functional independence, prevent or alleviate pain, and maintain health-related quality of life with limited burden to patients. ${ }^{1-3}$

The skeleton is the most common site for metastasis among patients with advanced cancer, resulting in loss of structural integrity of the bone and significant patient morbidity. ${ }^{4}$ Bone metastases reduce weight-bearing capabilities of bone and frequently lead to skeletal complications such as pathologic fractures, spinal cord compression, and radiation or surgery to the bone, ${ }^{1}$ collectively referred to as skeletalrelated events (SREs).

Treatment options for bone metastases include modalities such as radiotherapy, orthopedic surgery, bone-targeting pharmaceuticals, and antitumor therapy. Zoledronic acid (ZA), marketed as Zometa ${ }^{\circledR}$ (Novartis, Basel, Switzerland), is 
commonly prescribed to delay SREs in patients with bone metastases. In a pivotal Phase III ZA clinical trial, decreased infusion time was found to be associated with decreased renal safety. ${ }^{5}$ Therefore, $\mathrm{ZA}$ is administered intravenously every 3 to 4 weeks $^{6}$ over a minimum of 15 minutes to reduce the risk of clinically significant deterioration in renal function. ZA's infusion time has been cited as an advantage over other bisphosphonates (eg, pamidronate), ${ }^{7,8}$ which typically require 2 to 3 hours for infusion. In clinical practice, however, administration time for ZA is notably longer than the 15-minute minimum specified on the label. Additionally, due to the risk of renal toxicity, renal monitoring is recommended before each intravenous (IV)

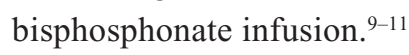

Longer administration time associated with ZA and the potential need for a separate visit for serum creatinine testing for renal monitoring may add substantially to the burden to patients, caregivers, and healthcare providers. However, limited data have been published regarding the time burden associated with cancer care. A study assessing the time burden on patients receiving care for colorectal cancer estimated that patients spent, on average, 432 hours seeking medical services (such as office visits, emergency room visits, chemotherapy, radiation therapy, hospitalization, and outpatient surgery) during their final 12 months of life. ${ }^{12}$ This estimate included time spent travelling to and from the clinic, waiting at the clinic, and receiving care. Additional burden associated with drug administration and visits to the clinic can add substantially to the already high disease burden associated with advanced cancer. In addition, cancer care can also significantly add to the caregivers' burden. ${ }^{12}$ A study estimating time costs associated with informal caregiving for cancer survivors concluded that the amount of caregiver time spent was highest for patients with metastatic disease compared to patients with local or regional cancers.

A handful of time and motion studies on treatment of bone metastases have been published. ${ }^{7,8,13-15}$ One study was published in Italian, ${ }^{14}$ and another was available as an abstract only. ${ }^{15}$ A study in the UK at three hospitals reported that while intravenous (IV) ZA infusion, on average, took 18 minutes, patients spent an average of 98 minutes at the administration unit. ${ }^{13}$ The single peer reviewed manuscript from a US-based study reported data from three sites, each with two patients per site where only one patient received IV ZA. This small study estimated that administration time for ZA averaged 66 minutes, of which 25 minutes was accounted for by infusion time and the remaining 41 minutes by ancillary tasks. ${ }^{7}$
The applicability of these studies to current clinical practice in the US is limited as they either included few patients, were conducted outside of the US, or were conducted prior to the inclusion of renal monitoring guidelines which recommend serum creatinine testing before IV bisphosphonate administration.

As the majority of patients with breast or prostate cancer and bone metastases do not receive ZA infusion and chemotherapy infusion on the same day, the primary objective of this analysis was to delineate the overall time associated with administration of IV ZA in patients with bone metastases secondary to breast or prostate cancer who did not receive chemotherapy on the same day. A secondary objective was to quantify the mean time spent by healthcare professionals in the preparation or administration of ZA.

\section{Methods}

This time and motion study was conducted at seven oncology clinics in the US and measured time associated with administration of IV ZA in patients with bone metastasis secondary to breast or prostate cancer.

\section{Eligibility criteria}

Patients were eligible to be included in this study if they were 18 years of age or older, were able to read and write English, and had a confirmed diagnosis of bone metastasis secondary to breast or prostate cancer. Each patient was observed only once and subsequent visits by the same patient for ZA infusions were not included. In addition, patients who were participating in a clinical trial of an investigational new drug to treat bone metastasis were excluded from the study. Eligibility of the sites to participate in this study was based on prior clinical research experience and availability of a study coordinator on site. In addition, sites were also required to be administering ZA to 25 to 50 patients per month to be considered eligible to participate in this study. In order to delineate the time burden specifically associated with ZA, patients who visited the clinic to receive IV ZA but were not scheduled to receive chemotherapy on the same day were identified. Data for patients who received chemotherapy in conjunction with ZA administration during the same infusion visit have been reported previously. ${ }^{16}$

Study protocol, informed consent, and all other studyrelated materials were approved by institutional review boards at each participating site prior to the start of data collection. Written informed consent was obtained from all patients prior to enrollment. 


\section{Observer training}

Data were entered in the observer form by six trained observers. Observers were trained by observing and timing five mock videotaped sessions of ZA administration. Observer inter-rater reliability was assessed by calculating the intraclass correlation coefficient (ICC). All observers were required to obtain an ICC of at least 0.7 or greater to be qualified to observe patients in this study. ${ }^{16}$ In addition, interabstractor reliability was also evaluated by having two independent trained observers time the infusion activities for $10 \%$ of the patients.

\section{Data collection}

Data were collected using an observer form, site questionnaire, and a case report form. The observer form was used to collect time and motion data associated with ZA administration. This form collected total administration time for each task, as well as time spent by each healthcare provider during the administration of ZA. A site questionnaire was used to record details about each site, such as geographic location, number of staff, number of patient visits per day, staff experience, number of infusion chairs, third-party payer distribution, and site-specific variable and fixed costs. Case report forms were used to collect patient information such as demographics, medical history, comorbidities, renal function assessment (date and location), and concomitant medication use.

Time and motion data associated with individual activities such as preinfusion tasks (vital signs, blood draw, and physical exam), ZA preparation, infusion tasks (hydration and ZA infusion), and follow-up activities (eg, removal of IV access line, recording of blood pressure, assessment for adverse drug reactions, patient counseling, prescribing medications) were recorded by trained observers on the observer form using a calibrated stop watch. For tasks which occurred concurrently, the task that involved direct contact with the patient was preferentially recorded and, for tasks that could not be observed, time was assigned based on the shortest recorded time for other patients receiving such a task.

\section{Statistical analysis}

No formal hypotheses were tested. Descriptive summaries for all variables were provided, including mean, median, and standard deviation (SD) for continuous variables, and frequency distribution and percentages for categorical variables. Data were tabulated in subgroups by cancer type (breast or prostate cancer).

\section{Results}

\section{Clinic details}

Data were collected from seven clinics in the US, with a mean clinic size of 10,086 square feet and approximately five examination rooms. ${ }^{16}$ On average, clinics were open for 39 hours per week and staffed with two full-time medical oncologists. Each clinic provided IV ZA treatment to an average of 42.4 patients per month and the mean treatment duration was 17.6 months. An ICC value of 0.94 for pre-study observer training and of $>0.99$ for dual abstractions by two independent abstractors was achieved in this study. ${ }^{16}$

\section{Patient characteristics}

A total of 68 patients were enrolled, 42 with breast cancer and 26 with prostate cancer. Data for 24 breast and 15 prostate cancer patients who received ZA during their regularly scheduled office visit and did not receive chemotherapy are reported here.

Mean age for breast cancer patients was 60.6 (SD 12.6) years and for prostate cancer patients was 73.9 (SD 8.5) years. Breast cancer patients had been diagnosed with bone metastases for a mean of 2.2 (SD 1.5) years and prostate cancer patients had been diagnosed for a mean of 2.0 (SD 2.7) years. Baseline demographic and clinical characteristics are summarized in Table 1 by cancer type. The most common comorbid conditions observed in this sample included musculoskeletal comorbidities (breast cancer: $58 \%$; prostate cancer: $40 \%$ ), cardiovascular and respiratory comorbidities (breast cancer: 25\%; prostate cancer: $73 \%$ ), and endocrine-related comorbidities (breast cancer: 45\%; prostate cancer: $27 \%$ )

Table I Demographic information by cancer type

\begin{tabular}{|c|c|c|c|}
\hline & $\begin{array}{l}\text { Breast cancer } \\
(N=24)\end{array}$ & $\begin{array}{l}\text { Prostate cancer } \\
(N=15)\end{array}$ & $\begin{array}{l}\text { Total } \\
(N=39)\end{array}$ \\
\hline \multicolumn{4}{|l|}{ Age (years) } \\
\hline Mean( SD) & $60.6(12.6)$ & $73.9(8.5)$ & $65.7(12.9)$ \\
\hline$\geq 65 \mathrm{n}(\%)$ & $9(37.5 \%)$ & $13(86.7 \%)$ & $22(56.4 \%)$ \\
\hline \multicolumn{4}{|l|}{ Gender n (\%) } \\
\hline Male & $0(0.0 \%)$ & $15(100.0 \%)$ & $15(38.5 \%)$ \\
\hline \multicolumn{4}{|l|}{ Race/ethnicity n (\%) } \\
\hline White or caucasian & $21(87.5 \%)$ & $15(100.0 \%)$ & $36(92.3 \%)$ \\
\hline Hispanic or latino & $3(12.5 \%)$ & $0(0.0 \%)$ & $3(7.7 \%)$ \\
\hline \multicolumn{4}{|c|}{ Years since cancer diagnosis } \\
\hline Mean (SD) & $7.3(5.1)$ & $7.8(6.8)$ & $7.5(5.7)$ \\
\hline \multicolumn{4}{|c|}{ Years since onset of metastatic bone disease } \\
\hline Mean (SD) & $2.2(1.5)$ & $2.0(2.7)$ & $2.1(2.0)$ \\
\hline \multicolumn{4}{|c|}{ Years from cancer diagnosis to onset of metastatic bone disease } \\
\hline Mean (SD) & $5.1(4.6)$ & $5.8(6.6)$ & $5.4(5.4)$ \\
\hline
\end{tabular}


Table 2 Renal assessment

\begin{tabular}{|c|c|c|c|}
\hline & $\begin{array}{l}\text { Breast cancer } \\
(N=24)\end{array}$ & $\begin{array}{l}\text { Prostate cancer } \\
(N=15)\end{array}$ & $\begin{array}{l}\text { Total } \\
(\mathbf{N}=39)\end{array}$ \\
\hline $\begin{array}{l}\text { Renal function } \\
\text { assessed n (\%) }\end{array}$ & $15(62.5 \%)$ & $13(86.7 \%)$ & $28(71.8 \%)$ \\
\hline $\begin{array}{l}\text { Assessments done } \\
\text { during a separate } \\
\text { visit }^{\mathrm{a}, *} \mathrm{n}(\%)\end{array}$ & $6(40 \%)$ & 5 (38.4\%) & II (39.2\%) \\
\hline $\begin{array}{l}\text { On-site laboratory } \\
\text { assessment of those } \\
\text { whose renal function } \\
\text { was assessed) } n(\%)\end{array}$ & $9(60 \%)$ & $8(6 \mid .5 \%)$ & $17(60.7 \%)$ \\
\hline
\end{tabular}

Note: a,*Of those whose renal function was assessed.

Secondary to renal toxicity, the Zometa label specifies that renal function should be assessed prior to each infusion. Renal function was assessed for $63 \%$ of breast and $87 \%$ of prostate cancer patients prior to infusion. Table 2 summarizes patient renal assessment by cancer type.

\section{Administration of ZA}

Administration time by each activity and cancer type is summarized in Table 3. Mean ZA administration time was 72.1(SD 47.1) minutes for breast cancer, 65.0 (SD 32.5) minutes for prostate cancer, and 69.4 (SD 41.8) minutes for both cancer groups combined. The median overall administration time for breast and prostate cancer combined was 60 minutes. Mean ZA infusion time was 33.9 (SD 15.1) minutes for breast cancer, 29.4 (SD 12.1) minutes for prostate cancer, and 32.2 (SD 14) minutes for both cancers combined. Approximately $58 \%(n=14)$ of breast cancer and $60 \%(n=9)$ of prostate cancer patients received hydration prior to ZA administration. Mean infusion time for hydration was 33.2 (SD 29.6) minutes for breast cancer, 36.6 (SD 26.4) minutes for prostate cancer, and 34.6 (SD 27.8) minutes for both cancers combined.
The mean time spent by healthcare providers for IV infusion of ZA was 16.4 (SD 21.0) minutes for registered nurses, 10.7 (SD 20.2) minutes for nurse practitioners, and 8.1 (SD 6.5) minutes for physicians (Table 4). Pharmacists' time was generally not captured as ZA preparation occurred simultaneously with other activities.

\section{Discussion}

$\mathrm{ZA}$ is administered to patients with bone metastasis secondary to advanced cancer over a minimum of 15 minutes to reduce the risk of renal toxicity. This 15-minute minimum infusion time has been cited as an advantage of ZA over other bisphosphonates that require longer infusion time. ${ }^{13}$ In clinical practice, however, overall administration time for ZA may be notably greater than the minimum 15 minutes recommended on the label, and has been found to average from 60 to 98 minutes, ${ }^{7,13}$ due to additional activities related to preinfusion, drug preparation, drug infusion, and followup. This extended administration time can burden patients, healthcare personnel, and hospital or clinic resources.

Unlike previous studies that were conducted during clinical trials and/or included few patients,,${ }^{78}$ this time and motion study was conducted when patients made regularly scheduled clinic visits to receive IV ZA, and recruited a much larger patient group for both breast and prostate cancer. As the patients in this analysis were not scheduled to receive concomitant chemotherapy, the time estimates reported here are directly attributable to ZA administration. Research has shown that majority of patients receiving ZA do not receive chemotherapy on the same day, with a US claims database analysis demonstrating that only $30 \%$ of patients receive both ZA and chemotherapy on the same day. Consequently, time and motion results for patients receiving IV ZA without sameday chemotherapy administration are relevant to the majority

Table 3 Administration time by cancer type

\begin{tabular}{|c|c|c|c|c|c|c|}
\hline \multirow[t]{3}{*}{ Activity/task } & \multicolumn{2}{|c|}{ Breast cancer $(N=24)$} & \multicolumn{2}{|c|}{ Prostate cancer $(\mathbf{N}=\mid \mathbf{5})$} & \multicolumn{2}{|c|}{ Total $(\mathbf{N}=39)$} \\
\hline & n (\%) & Mean (SD) & n (\%) & Mean (SD) & n (\%) & Mean (SD) \\
\hline & & Time, minutes & & Time, minutes & & Time, minutes \\
\hline Pre-infusion & $24(100.0)$ & $12.5(24.8)$ & $15(100.0)$ & $10.6(7.4)$ & $39(100.0)$ & II.8 (I9.8) \\
\hline Vital signs & $24(100.0)$ & $6.6(2 \mathrm{I} .5)$ & $13(100.0)$ & $2.0(0.8)$ & 37 (94.8) & $5.0(17.4)$ \\
\hline Blood draw & $12(50.0)$ & $2.2(1.2)$ & $8(53.0)$ & $4.0(3.4)$ & $20(5 \mid .2)$ & $2.9(2.4)$ \\
\hline Physical exam & I $3(54.0)$ & $8.9(7.5)$ & $10(66.0)$ & $10.1(6.1)$ & $23(58.9)$ & $9.4(6.8)$ \\
\hline Zoledronic acid preparation & $24(100)$ & $2.1(I . I)$ & $15(100.0)$ & $1.6(I . I)$ & $39(100.0)$ & $1.9(I . I)$ \\
\hline Infusion & $24(100)$ & $55.4(31.4)$ & $15(100.0)$ & $51.4(30.9)$ & $39(100.0)$ & $53.9(30.8)$ \\
\hline Hydration & $14(58.0)$ & $33.2(29.6)$ & $9(60.0)$ & $36.6(26.4)$ & $23(58.9)$ & $34.6(27.8)$ \\
\hline Zoledronic acid & $24(100.0)$ & $33.9(15.1)$ & I5 (I00.0) & $29.4(12.1)$ & $39(100.0)$ & $32.2(14.0)$ \\
\hline Follow-up & $24(57.1)$ & $2.0(1.5)$ & I 5 (57.7) & $1.4(0.6)$ & $39(57.4)$ & I.8 (I.2) \\
\hline Total & $24(57.1)$ & 72.1 (47.I) & I5 (57.7) & $65.0(32.5)$ & $39(57.4)$ & $69.4(41.8)$ \\
\hline
\end{tabular}

Abbreviation: SD, standard deviation. 
Table 4 Time spent by each health care personnel during patient visit

\begin{tabular}{|c|c|c|c|c|c|c|}
\hline \multirow[t]{2}{*}{ Health care personnel } & \multicolumn{2}{|c|}{ Breast cancer $(\mathbf{N}=24)$} & \multicolumn{2}{|c|}{ Prostate cancer $(\mathbf{N}=15)$} & \multicolumn{2}{|c|}{ Total $(\mathbf{N}=39)$} \\
\hline & $\mathrm{n}^{\mathrm{a}}$ & $\begin{array}{l}\text { Total time, minutes } \\
\text { Mean (SD) }\end{array}$ & $\mathbf{n}^{\mathbf{a}}$ & $\begin{array}{l}\text { Total time, minutes } \\
\text { Mean (SD) }\end{array}$ & $\mathbf{n}^{\mathbf{a}}$ & $\begin{array}{l}\text { Total time, minutes } \\
\text { Mean (SD) }\end{array}$ \\
\hline Physician (MD) & 12 & $7.4 I(7.6 I)$ & 10 & $8.55(6.32)$ & 22 & $8.15(6.48)$ \\
\hline Registered nurse (RN) & 86 & $16.56(22.19)$ & 51 & $16.10(19.45)$ & 137 & $16.39(21.20)$ \\
\hline Nurse practitioner (NP) & 10 & $11.7(23.19)$ & 3 & $6.50(5.47)$ & 13 & $10.7(20.25)$ \\
\hline Pharmacist (eg, PharmD) ${ }^{b}$ & 4 & $2.34(0.21)$ & NA & NA & 4 & $2.34(0.21)$ \\
\hline Pharmacy technician (PT) ${ }^{\mathrm{b}}$ & 3 & $2.7(1.15)$ & 1 & 2.54 (NA) & 4 & $2.19(1.6)$ \\
\hline Other & 18 & $2.46(1.7)$ & 10 & $2.51(2.44)$ & 28 & $2.48(\mathrm{I} .48)$ \\
\hline
\end{tabular}

Notes: ${ }^{a}$ stands for number of observations and may include multiple observations for the same patient; ${ }^{b}$ time needed for some activities may not be recorded due to simultaneous occurrence.

Abbreviations: SD, standard deviation; NA, not applicable.

of patients with bone metastases who are treated with IV ZA. This study was conducted at multiple sites and can therefore be considered more representative of clinical practice in the US compared to previous studies. The observers employed for data collection were rigorously trained to ensure high data quality. Therefore, the time and motion methodology used in this study was more robust compared with previous studies.

Results from this study confirmed that ZA infusion combined with ancillary tasks associated with IV infusions resulted in a total administration time that was much longer (mean 69.4 minutes; median 60 minutes) than the minimum infusion time stated in the Zometa label. Further break down of time by activity found that on average, ZA infusion alone took 32.2 minutes, which is more than twice the minimum 15-minute infusion time recommended. In addition, as ZA and other bisphosphonates are risk factors for subsequent renal deterioration, hydration therapy is required for all patients receiving ZA. This study found that administration of hydration therapy significantly prolonged ZA administration time. Additionally, this study found that longer infusion time for IV ZA also required substantial time commitment per patient from multiple healthcare personnel (such as registered nurses, nurse practitioners, and physicians).

A limitation of this study is that only direct time data in the clinic were collected, so indirect time burden on patients in terms of lost work time or time for travel were not captured. Also, many patients made separate visits to the laboratory for serum creatinine testing and this additional time was not quantified in this study. Therefore, the overall time burden to patients and their caregivers is expected to be greater than that estimated in this study. Additionally, time for tasks that occurred simultaneously was not recorded separately, and could potentially lead to under-reporting of the time burden on healthcare professionals. In addition, as healthcare personnel administering IV ZA were aware that they were being observed and timed in relation to activities associated with ZA infusion, they may have demonstrated increased efficiency in their activities (ie, Hawthorne effect). ${ }^{17}$ Lastly, only sites that were administering ZA to 25 to 50 patients per month and had prior clinical research experience were eligible to participate in this study. Therefore, the results reported here may be generalized only to similar practices.

\section{Conclusion}

This time and motion study quantified the overall time required for IV ZA administration for patients with bone metastasis secondary to breast or prostate cancers, who received IV ZA infusion during a regularly scheduled infusion clinic visit. Overall, it was found that the mean time for ZA infusion, including hydration, was much higher than the minimum infusion time specified in the Zometa package insert. In addition to longer administration time, ZA infusion was also associated with a substantial time burden for healthcare providers. The results of this study demonstrate the need for more efficient therapeutic agents to decrease the burden associated with cancer care in patients with bone metastasis.

\section{Acknowledgments}

This study was funded by Amgen Inc, Thousand Oaks, CA. Editing assistance was provided by Amgen employee Vidya Setty, MPH, MBA.

\section{Disclosure}

All authors are employed by and hold stock/stock options with Amgen Inc, which funded this study.

\section{References}

1. Coleman RE. Bisphosphonates: clinical experience. Oncologist. 2004; 9 Suppl 4:14-27.

2. James ND, Bloomfield D, Luscombe C. The changing pattern of management for hormone-refractory, metastatic prostate cancer. Prostate Cancer Prostatic Dis. 2006;9(3):221-229.

3. Janjan N. Bone metastases: approaches to management. Semin Oncol. 2001;28(4 Suppl 11):28-34. 
4. Coleman RE. Clinical features of metastatic bone disease and risk of skeletal morbidity. Clin Cancer Res. 2006;12(20 Pt 2):6243s-6249s.

5. Saad F, Gleason DM, Murray R, et al. A randomized, placebo-controlled trial of zoledronic acid in patients with hormone-refractory metastatic prostate carcinoma. J Natl Cancer Inst. 2002;94(19):1458-1468.

6. Zometa ${ }^{\circledR}$ (zoledronic acid) [prescribing information]. East Hanover, NJ: Novartis Pharmaceuticals Corporation; 2008.

7. DesHarnais CL, Bajwa K, Markle JP, Timbie JW, Zacker C, Schulman KA. A microcosting analysis of zoledronic acid and pamidronate therapy in patients with metastatic bone disease. Support Care Cancer. 2001;9(7):545-551.

8. Dranitsaris G, Castel LD, Baladi JF, Schulman KA. Zoledronic acid versus pamidronate as palliative therapy in cancer patients: a Canadian time and motion analysis. J Oncol Pharm Prac. 2001;7(1):27-33.

9. Berenson JR, Hillner BE, Kyle RA, et al. American Society of Clinical Oncology clinical practice guidelines: the role of bisphosphonates in multiple myeloma. J Clin Oncol. 2002;20(17):3719-3736.

10. Hillner BE, Ingle JN, Berenson JR, et al. American Society of Clinical Oncology guideline on the role of bisphosphonates in breast cancer. American Society of Clinical Oncology Bisphosphonates Expert Panel. J Clin Oncol. 2000;18(6):1378-1391.

11. Hillner BE, Ingle JN, Chlebowski RT, et al. American Society of Clinical Oncology 2003 update on the role of bisphosphonates and bone health issues in women with breast cancer. J Clin Oncol. 2003;21(21):4042-4057.
12. Yabroff KR, Warren JL, Knopf K, Davis WW, Brown ML. Estimating patient time costs associated with colorectal cancer care. Med Care. 2005;43(7):640-648.

13. Barrett-Lee P, Bloomfield D, Dougherty L, et al. An audit to determine the time taken to administer intravenous bisphosphonate infusions in patients diagnosed with metastatic breast cancer to bone in a hospital setting. Curr Med Res Opin. 2007;23(7):1575-1582.

14. Schivazappa C, Baldini E, Cortesi E, Dauria G, Rolia F, Berto P. Cost analysis of tumor-induced osteolysis treated with intravenous zoledronic acid and pamidronate: a time-motion study. Recenti Prog Med. 2007;98(1):12-15.

15. Wardley A, Body JJ, Neary M. A time-in-motion study of oral ibandronate versus IV zoledronic acid for treatment of metastatic bone disease in breast cancer patients. J Clin Oncol. 2005;23(Suppl 1):6123.

16. Oglesby A, Sherif B, Odom D, Leahy M, Qian Y. Time and costs associated with preparing and administering zoledronic acid in patients with breast or prostate cancer and metastatic bone disease. Community Oncol. 2009;6(11):494-505.

17. Fletcher RW, Fletcher SW. Clinical epidemiology. The essentials. 4th ed. Baltimore: Lippincott Williams \& Wilkins; 2005.

\section{Publish your work in this journal}

Cancer Management and Research is an international, peer-reviewed open access journal focusing on cancer research and the optimal use of preventative and integrated treatment interventions to achieve improved outcomes, enhanced survival and quality of life for the cancer patient. The journal welcomes original research, clinical \& epidemiological

\section{Dovepress}

studies, reviews \& evaluations, guidelines, expert opinion \& commentary, case reports \& extended reports. The manuscript management system is completely online and includes a very quick and fair peerreview system, which is all easy to use. Visit http://www.dovepress.com/ testimonials.php to read real quotes from published authors. 